\title{
Efficient Low-Complexity Digital Predistortion for Power Amplifier Linearization
}

\author{
Siba Monther Yousif ${ }^{1,4}$, Roslina M. Sidek ${ }^{1}$, Anwer Sabah Mekki ${ }^{2}$, Nasri Sulaiman ${ }^{1}$, Pooria Varahram ${ }^{3}$ \\ ${ }^{1}$ Department of Electrical and Electronic Engineering, Universiti Putra Malaysia (UPM), Malaysia \\ ${ }^{2}$ Functional Devices Laboratory, Institute of Advanced Technology, Universiti Putra Malaysia (UPM), Malaysia \\ ${ }^{3}$ Department of Electronic Engineering, Maynooth University, Maynooth, Co. Kildare, Ireland \\ ${ }^{4}$ Department of Electronic and Communication Engineering, Al-Nahrain University, Baghdad, Iraq
}

\section{Article Info}

Article history:

Received Feb 9, 2016

Revised May 11, 2016

Accepted May 20, 2016

\section{Keyword:}

Digital Predistortion

Memory Effects

Memory Polynomial

Model Complexity

Power Amplifiers

\begin{abstract}
In this paper, a low-complexity model is proposed for linearizing power amplifiers with memory effects using the digital predistortion (DPD) technique. In the proposed model, the linear, low-order nonlinear and highorder nonlinear memory effects are computed separately to provide flexibility in controlling the model parameters so that both high performance and low model complexity can be achieved. The performance of the proposed model is assessed based on experimental measurements of a commercial class $\mathrm{AB}$ power amplifier by applying a single-carrier wideband code division multiple access (WCDMA) signal. The linearity performance and the model complexity of the proposed model are compared with the memory polynomial (MP) model and the DPD with single-feedback model. The experimental results show that the proposed model outperforms the latter model by $5 \mathrm{~dB}$ in terms of adjacent channel leakage power ratio (ACLR) with comparable complexity. Compared to MP model, the proposed model shows improved ACLR performance by $10.8 \mathrm{~dB}$ with a reduction in the complexity by $17 \%$ in terms of number of floating-point operations (FLOPs) and $18 \%$ in terms of number of model coefficients.
\end{abstract}

Copyright (C) 2016 Institute of Advanced Engineering and Science. All rights reserved.

Corresponding Author:

Siba Monther Yousif,

Department of Electrical and Electronic Engineering,

Universiti Putra Malaysia (UPM),

Serdang, 43400, Selangor, Malaysia.

Email: sibamonther2000@yahoo.com

\section{INTRODUCTION}

Power amplifier (PA) is a major source of nonlinearity in a communication system since it is often driven close to the saturation region to achieve high power efficiency. The nonlinearity includes out-of-band emission which causes adjacent channel interference and in-band distortion that degrades the bit error rate performance. In modern high speed communications, transmission schemes with high spectral efficiency such as Orthogonal Frequency Division Multiplexing (OFDM) and Wideband Code Division Multiple Access (WCDMA) are more sensitive to PA nonlinearity and memory effects. This issue can be solved by backing-off the operating region of the PA into a linear mode at the expense of the degradation of the power amplifier efficiency. To overcome the conflict between the power efficiency and the linearity of the power amplifier, a linearization technique is required. One of the most cost-effective linearization techniques is the digital predistortion (DPD) [1].

Many DPD structures have been presented in the literature, containing the Volterra-based models [2]-[6], the polynomial-based models [7], the neural-network models [8],[9], and the LUT-based models [10],[11]. Even though the Volterra model is generally the most accurate structure in compensating the nonlinearity with memory effects of the power amplifier, it is mainly restricted to compensate mild 
nonlinearity with memory effects of the PA. This is because of its high complexity in extracting Volterra kernels. Therefore, several special cases of Volterra model were proposed, such as the dynamic deviation reduction model [12].

However, high complexity DPD is undesirable because it leads to high power consumption and long-time delay due to intensive processing. Moreover, the main justification for the DPD technique is to gain more power-efficient PA, which is the most power consuming device in transmitters [13]-[15]. Therefore, it is essential that the power saved, by using DPD, is not spent on a high complexity DPD algorithm. Indeed, the MP model proposed in [2] is well-known for PA linearization. This model compensates for nonlinearities with memory effects using considerably lower model dimensions than the models reported in [4]. However, the linearity performance of the PA using the MP model is generally lower than the performance when using the models presented in [3],[4]. Therefore, achieving high linearity performance and simultaneously minimizing the DPD model complexity is crucial.

In this paper, a low-complexity DPD model is proposed and experimentally validated for linearizing power amplifiers with memory effects. The proposed model is constructed by separating the linear from the nonlinear memory effects to enhance linearization. The low-order nonlinear memory effect is then separated from its high-order terms to reduce the model computational complexity. Consequently, this algorithm will provide flexibility in controlling the dimensions of the model that can improve the linearity performance while reducing the computational complexity of the DPD model. Therefore, the main contribution of this paper is that the proposed model gives a better experimentally adjacent channel leakage power ratio (ACLR) performance than the MP model [2] with a considerable reduction in the model computational complexity. Moreover, the experimental results show that the proposed model outperforms the DPD model with singlefeedback [3] in terms of ACLR performance with a comparable model complexity.

\section{MODEL DESCRIPTION}

In this section, the memory polynomial model is presented and the proposed model with its identification algorithm is clarified.

\subsection{Memory Polynomial Model}

The baseband predistorter can be modelled using the MP model, which is a good model as considered in [2], as shown in Equation (1):

$$
z(n)=\sum_{k=1}^{K} \sum_{Q=0}^{Q} \epsilon_{k q} x(n-q)|x(n-q)|^{k-1}
$$

where $z(n)$ and $x(n)$ are the complex output and input signals of the MP predistorter model, respectively. $c_{\mathrm{n} q}, K$, and $Q$ are the model coefficients, nonlinearity order, and memory length, respectively.

In [2], the MP model, which was used as a digital predistorter, offers a good trade-off between performance and complexity. It has a good advantage since its parameters (i.e. the MP's coefficients) can easily be extracted using least square solutions with an indirect learning architecture proposed in [16] as shown in Figure 1. However, the MP model uses the same high nonlinearity order in all of the memory branches, which results in an oversized model and an increase in the computational complexity of its model.

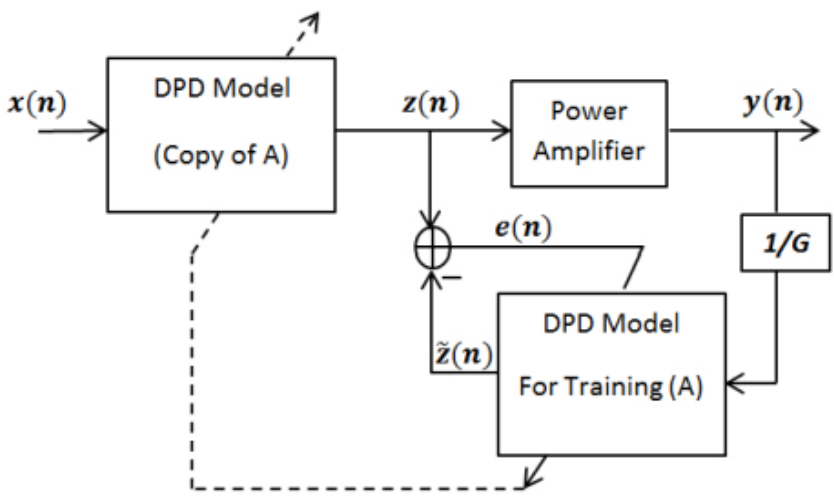

Figure 1. Indirect learning architecture 


\subsection{Proposed Model}

To reduce the complexity of the DPD model and enhance the compensating performance of the nonlinearities with memory effects of the power amplifier, a DPD model is derived from the Volterra-series model represented in complex baseband [17] as shown in Equation (2):

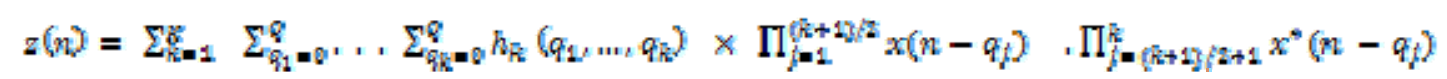

where $z(n)$ and $x(n)$ are the complex baseband output and input signals of the Volterra-series predistorter, respectively. $K$ is the nonlinearity order, $Q$ is the memory depth, $h_{k}\left(q_{L}, \ldots, q_{k}\right)$ are the $k$ th-order Volterra kernels, and the symbol denotes the complex conjugate operator.

If the Volterra kernels are equal to zero except along the diagonal where

only $q_{1}=q_{2}=m=q_{k}=q$ are considered, this will reduce the model complexity without significant reduction in the linearity performance. Thus, the expression in Equation (2) is reduced to Equation (3):

$$
z(n)=\sum_{k=1}^{*} \sum_{q=0}^{q} h_{k}(q, q, \ldots, q) x(n-q)|x(n-q)|^{\hat{k}-1}
$$

By combining the terms of the linear memory effects (i.e. when $k=1$ ) and separating them from the other terms, which represent the dynamic nonlinearity effects. Consequently, the linear memory effects can be efficiently compensated as shown in Equation (4):

$$
z(n)=\sum_{q=0}^{Q}\left[h_{1}(q) x(n-q)+\sum_{k=2}^{*} h_{k}\left(q \ldots \ldots, q_{k-1}\right) x(n-q)|x(n-q)|^{\bar{k}-1}\right]
$$

Then, for properly controlling the compensation of the nonlinearity with memory effects and reducing at the same time the computational complexity of the proposed model, the effects of the dynamic low-order are split from the high-order nonlinearity effects by sorting out the nonlinearity terms of $k=2$ from the higher nonlinearity order as illustrated in Equation (5):

$$
\begin{aligned}
& z(n)=\sum_{q-a}^{q}\left[h_{1}(q) x(n-q)+h_{2}(q, q) x(n-q)|x(n-q)|+\sum_{k-q}^{*} h_{k}\left(q, \ldots, q_{k-2}\right) x(n-\right. \\
& \left.q)|x(n-q)|^{k-1}\right]
\end{aligned}
$$

By changing the model coefficients $h_{1}(q), h_{2}(q, q)$, and $h_{i}\left(q \ldots \ldots q_{i}\right)$ to $a_{1 m}, a_{n i}$, and $x_{h q}$ respectively, the proposed method can be expressed as in Equation (6):

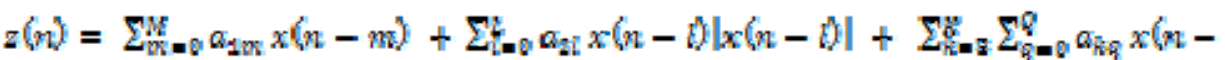

$$
\begin{aligned}
& \text { q) }|x(n-q)|^{i}-1
\end{aligned}
$$

where $a_{1 \mathrm{~m}}$ and $a_{2 i}$ are the complex coefficients of the first and second branches of the proposed model, respectively, and the $a_{h a}$ values include the complex model coefficients of the third branch. $M, L$, and $Q$ represent the memory depth for the first, second, and third branches, respectively, and $K$ denotes the nonlinearity order for the third branch. It is worth noting that the third term starts with nonlinearity order $k=3$ to avoid redundancy with the first and second terms.

As modern wireless systems utilize wider bandwidths with higher speed, the design for an accurately DPD model must take into account the linear memory effects and the dynamic nonlinearities. Thus, the proposed predistorter shown in Figure 2 has an important property, which is separating the purely linear memory effects (represented in the first branch) from the low-order nonlinearity dynamic one (considered in the second branch) and finally adds these branches to the high-order nonlinearity memory effects branch. Consequently, the proposed model provides an efficient way to present an effective distortion compensation approach for power amplifier linearization. Moreover, the proposed model also allows for more flexibility in modelling the memory effects in which the model dimensions of each branch are controlled separately, which reduces the model complexity while enhancing the linearity performance of PAs. 


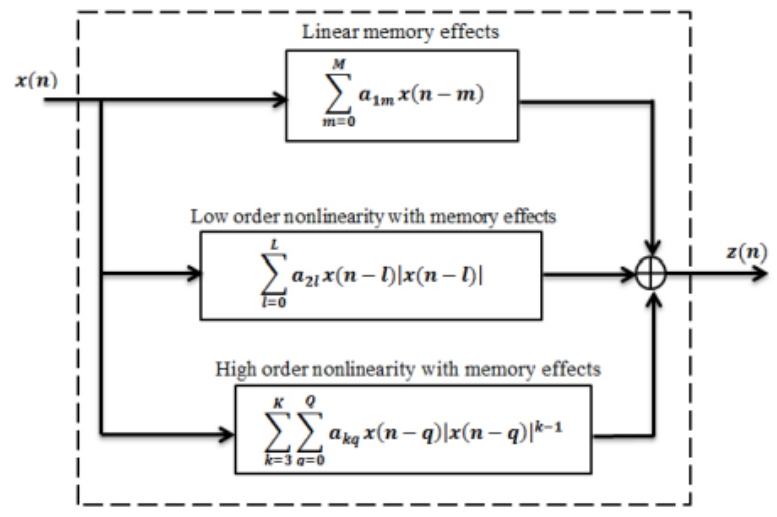

Figure 2. Basic architecture of the proposed model

\subsection{Model Identification Procedure}

The proposed predistorter has the property of linearity in model parameters as shown in Equation (6), which means that the model output is linear with its coefficients, since it was derived from Volterraseries model. Hence, the coefficients of the proposed model can be extracted in a direct way using the least squares (LS) technique. The identification of the proposed model is an offline procedure and all of the branches of Equation (6) are identified simultaneously as shown in Equation (7):

$$
z=E w
$$

where the $z$ vector is the output of the three dynamic branches based on Equation (6), the $U$ matrix includes the basis functions of the three polynomial branches, and the $w$ vector contains the coefficients of the proposed model. The vectors $z$ and $w$ are defined in Equations (8) and (9) respectively, where $N$ is the input samples length used for the identification:

$$
\begin{aligned}
& z=[z(0), z(1), \ldots, z(w-1)]^{7}
\end{aligned}
$$

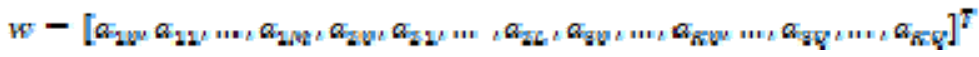

The matrix $v$ is analysed into sub-matrices as shown in Equation (10):

$$
U=\left[\begin{array}{lll}
U_{p 1} & U_{p 2} & U_{p g}
\end{array}\right]
$$

where the sub-matrices, $U_{p_{1}}, U_{p_{2}}$, and $v_{p g}$, are composed from the basis functions of the linear memory effects, low-order nonlinearity with dynamic effects, and dynamic high-order nonlinearity branches, respectively. The indirect learning architecture is used for extracting the coefficients of the proposed model as shown in Figure 1. Accordingly, a new sequence is defined in Equation (11):

$$
g(n)=\frac{x[n]}{\theta}
$$

where $g(n)$ is the complex baseband input signal of the predistorter during identification process, $y(n)$ is the complex baseband output signal of the PA, and $\boldsymbol{G}$ is the gain of the linearized PA. The vector $\boldsymbol{u}$ can be expressed in Equation (12):

$$
u=\left[g(0), g(1), \ldots, g(N V-11]^{2}\right.
$$

The sub-matrices $U_{p 1}, U_{p 2}$, and $U_{p g}$ are expressed in Equations (13), (14), and (15) respectively:

$$
\begin{aligned}
& U_{p 1}=[u(n), u(n-1), \ldots, u(n-M)]
\end{aligned}
$$

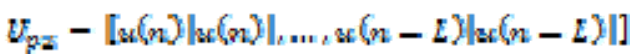


$U_{p S}=\left[u(n), u(n)|u(n)| \ldots, u(n)|u(n)|^{k-1}, \ldots, u(n-Q), \ldots, u(n-Q)|u(n-Q)|^{k-1}\right]$ (15)

Finally, the coefficients of the proposed model in Equation (6) can be determined using leastsquares solution for Equation (7) as shown in Equation (16), where $(.)^{H}$ represents complex conjugate transpose:

$$
w=\left(U^{H} U\right)^{-1} U^{H} z
$$

\section{EXPERIMENTAL SETUP}

To demonstrate the linearization ability of the proposed predistorter, measurements was performed using the experimental setup shown in Figure 3. It consists of a personal computer (PC), Agilent - EXG vector signal generator N5172B, Agilent - PXA signal analyzer N9030A, ATM attenuator - PNR AV08430, and PA under test. The PC contains three software, which are Agilent SystemVue 2015.01, Matlab 2014a, and Agilent 89600 VSA software. The complex input baseband signal was generated in Matlab. Then, by using SystemVue simulator, this signal was downloaded, through Local Area Network (LAN), into the EXG in order to excite the PA under test by the RF input signal. Then, the RF output from the power amplifier was attenuated by $10 \mathrm{~dB}$ and received by the PXA. This PXA was utilized to down-convert and demodulate the RF output signal to baseband signal cooperating with the VSA 89600 software. Then, the baseband input and output waveforms were used to extract the coefficients of the predistortion functions in Matlab. After that, synthesizing the predistorted signal and downloading this signal into the EXG were carried out using SystemVue software.

The PA under test used was the HMC-C074 single stage class AB power amplifier, from Hittite Microwave Corporation, which provides $13 \mathrm{~dB}$ gain and $29.5 \mathrm{dBm}$ output power at $1 \mathrm{~dB}$ gain compression and can operate from $10 \mathrm{MHz}$ to $6 \mathrm{GHz}$. The PA was operated at $2.14 \mathrm{GHz}$ with an input peak power backoff of $1 \mathrm{~dB}$ and tested under 5-MHz bandwidth of a single-carrier WCDMA signal with peak-to-average power ratio (PAPR) of $8.7 \mathrm{~dB}$ and the signal was sampled at $25 \mathrm{MHz}$.

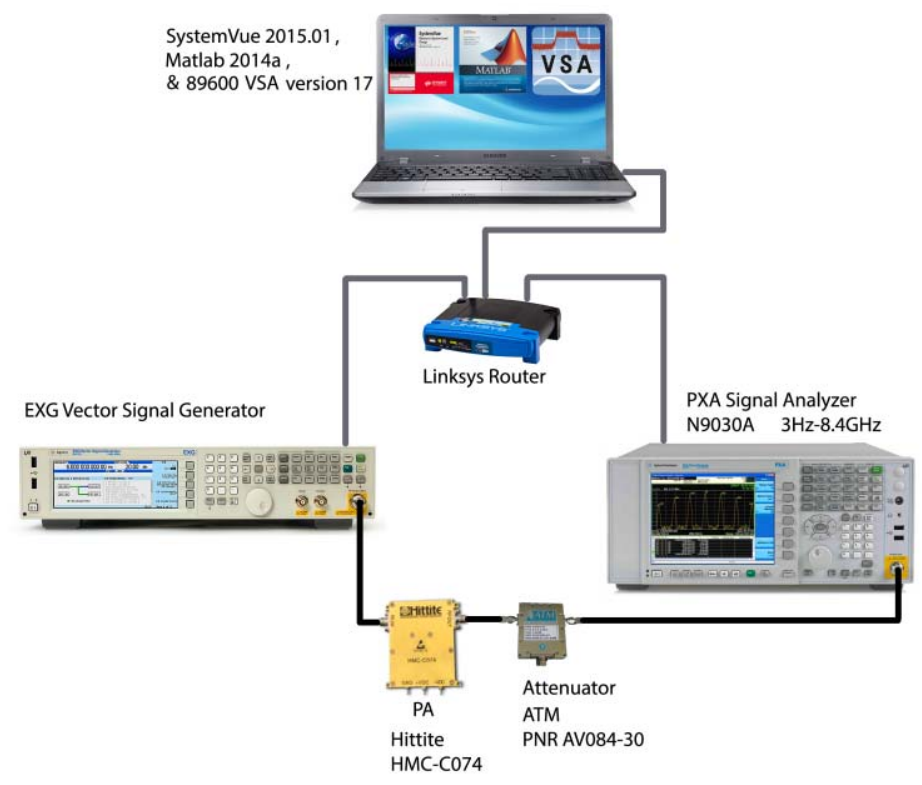

Figure 3. Measurement setup used for the proposed DPD validation

The complex input and output baseband waveforms, from the real PA, containing 20000 samples were utilized to extract the coefficients of the MP model according to Equation (1) and the proposed model based on Equation (6) using the training path shown in Figure 1. The dimensions of the models were appropriately selected to make a suitable trade-off between complexity and accuracy. The computational complexity will be discussed in section 5 and the model accuracy of the proposed and MP models were evaluated using the normalized mean squared error (NMSE) criterion, which is described in Equation (17):

IJECE Vol. 6, No. 3, June 2016 : $1096-1105$ 


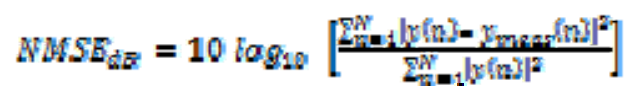

where $y(n)$ is the desired output waveform, $y_{\text {neas }}(n)$ is the measured output waveform, and $N$ is the number of samples utilized in these output waveforms.

The calculated NMSE and model dimensions of the proposed and MP models are listed in Table 1 as well as the NMSE and model dimensions of the model proposed in [3]. From Table 1, it can be observed that the proposed model has higher accuracy than the accuracy of the MP model by $2.1 \mathrm{~dB}$ and slightly comparable accuracy with respect to the proposed model in [3].

Table 1. Comparison of model dimensions and NMSE of different DPD models

\begin{tabular}{ccc}
\hline DPD model & Model dimensions & NMSE $(\mathrm{dB})$ \\
\hline MP [2] & $(K, Q)=(7,3)$ & -34.7 \\
DPD model [3] & $\left(K_{\text {odd order }}, Q\right)=(11,3)$ & -37.1 \\
Proposed model & $(M)(L)(K, Q)=(3)(3)(7,2)$ & -36.8 \\
\hline
\end{tabular}

\section{MEASUREMENT RESULTS}

In order to assess the effectiveness of the proposed predistorter, the PA was linearized using the well-known MP model based on Equation (1) with $\mathrm{Q}=3$ and $\mathrm{K}=7$, and the proposed model based on Equation (6) with $\mathrm{M}=3, \mathrm{~L}=3, \mathrm{~K}=7$, and $\mathrm{Q}=2$. The measured output spectra of the power amplifier before and after applying the proposed and MP DPDs are shown in Figure 4 and listed in Table 2 with the measured results of the DPD model illustrated in [3]. Before applying DPD, the ACLR of the PA output was $-40.5 \mathrm{dBc}$ while after applying MP model, the compensation of dynamic nonlinearity was $-51.3 \mathrm{dBc}$. Moreover, the model presented in [3] and listed in Table 2 has more reduction in ACLR than the MP model where -57 dBc was obtained. However, further ACLR improvement can be achieved when using the proposed model and the ACLR performance was $-62.1 \mathrm{dBc}$. Therefore, the experimental results illustrate a better linearization performance using the proposed model than the performance achieved by the MP model and the model proposed in [3] by $10.8 \mathrm{~dB}$ and $5 \mathrm{~dB}$, respectively. This achievement was obtained because of addressing the linear memory effects and separately compensating the effects of the low-order and the high-order nonlinear memory effects in the proposed model.

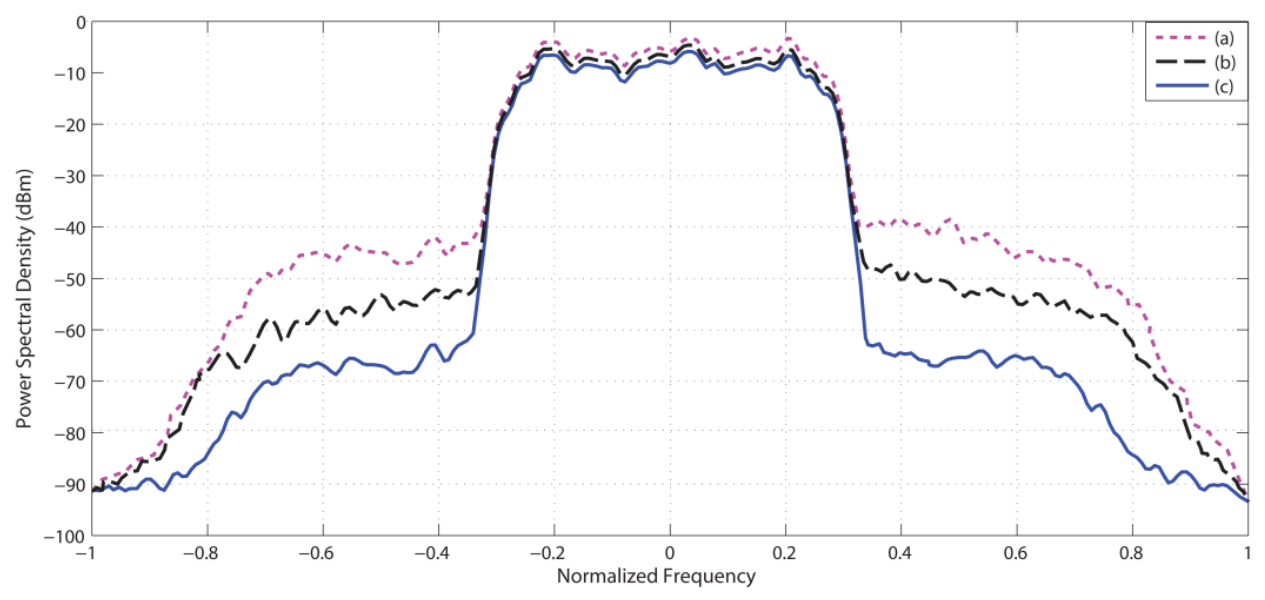

Figure 4. Measured spectra of the PA with 5-MHz WCDMA signal excitation. (a) Without DPD (b) With MP model $(\mathrm{K}=7$ and $\mathrm{Q}=3)$. (c) With proposed model $(\mathrm{M}=3, \mathrm{~L}=3, \mathrm{~K}=7$, and $\mathrm{Q}=2)$

Table 2. Comparison of ACLR performance of the PA

\begin{tabular}{ccc}
\hline DPD model & \multicolumn{2}{c}{ ACLR (dBc) } \\
& $-5 \mathrm{MHz}$ & $+5 \mathrm{MHz}$ \\
\hline Without DPD & -40.5 & -41 \\
MP model [2] & -51.3 & -50.5 \\
DPD model [3] & -57 & -56 \\
Proposed model & -62.1 & -61 \\
\hline
\end{tabular}

Efficient Low Complexity Digital Predistortion for Power Amplifier Linearization (Siba Monther Yousif) 
To further demonstrate the effectiveness of the proposed predistorter, dynamic AM/AM and $\mathrm{AM} / \mathrm{PM}$ characteristics of the power amplifier, driven by a single-carrier WCDMA signal with 5-MHz bandwidth, before and after applying the proposed and MP predistorters are shown in Figure 5 and Figure 6, respectively. From these figures, the dispersions and bending of the PA characteristics are shown before applying the proposed and MP models due to the electrical memory effects and nonlinearities, respectively. Figure 5 shows that the dispersions with bending of the actual PA characteristics are better compensated after applying the proposed model than the MP model. While, Figure 6, illustrates that the linearization capability on the dynamic AM/PM characteristics of the real PA of both the MP and proposed predistorters is mainly the same.

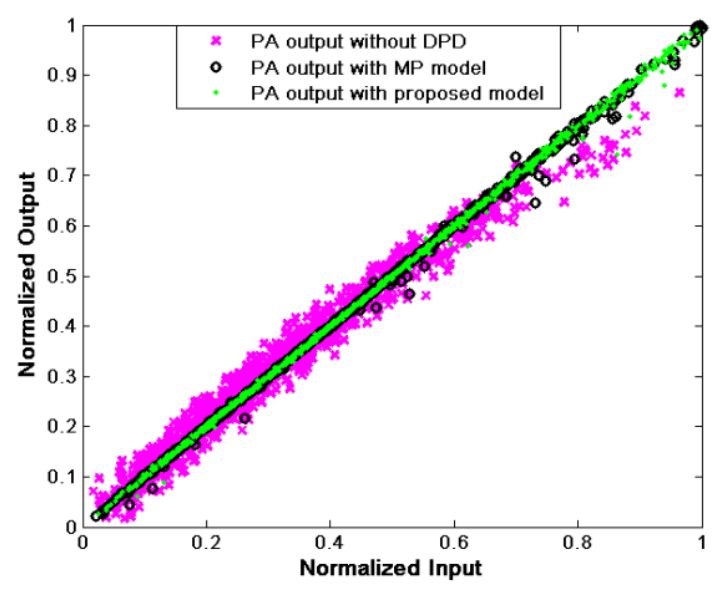

Figure 5. Dynamic AM/AM characteristics of the real PA driven by 5-MHz WCDMA signal

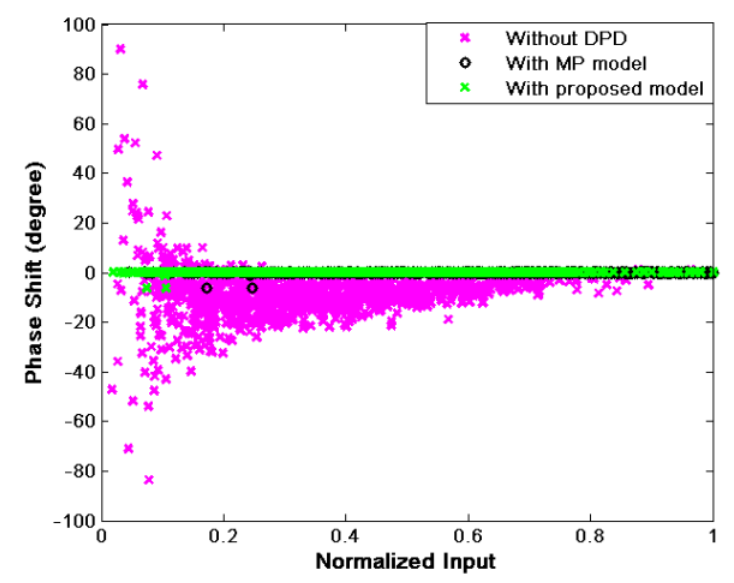

Figure 6. Dynamic AM/PM characteristics of the real PA driven by 5-MHz WCDMA signal

\section{COMPUTATIONAL COMPLEXITY ANALYSIS}

To evaluate the proposed predistorter in terms of computational complexity reduction, the model complexity of the proposed algorithm is determined and compared with the computational complexity of both the MP model [2] and the DPD model presented in [3]. In [18], it has been demonstrated that considering even order nonlinearities give a better model performance than using only odd order terms. Therefore, both even and odd orders of nonlinearities in the proposed model are considered in this comparison.

The complexity of the DPD models is evaluated based on the number of floating-point operations (FLOPs) and the number of model coefficients, as in [14],[19]. FLOPs are an actual measure for model complexity that gives the number of subtractions, additions, and multiplications used when the output of the DPD model is calculated. As explained in [19], the number of FLOPs required in each DPD model includes 
FLOPs during the construction of the basis functions and FLOPs when these basis functions are filtered by the model coefficients.

The number of FLOPs and model coefficients of the MP, DPD model presented in [3], and proposed model are reported in Table 3. The MP model has equal nonlinearity order in all of the memory branches and MP dimensions were set to 7 and 3 for $\mathrm{K}$ and Q, respectively. Consequently, this results in 244 FLOPs and 28 coefficients according to Equation (1). Conversely, the use of three dynamic branches in the proposed predistorter makes it possible to reduce the memory depth of the third branch to be applied with flexibility in the other two branches as shown in Figure 2. Accordingly, the dimensions of the proposed predistorter were set to 3, 3, 7, and 2 for M, L, K started from 3, and Q, respectively. Thus, the FLOPs and number of coefficients are reduced to 204 and 23, respectively, based on Equation (6). Therefore, as shown in Table 3, it can be concluded that the proposed model has computational complexity reduction of approximately $17 \%$ in terms of FLOPs and 18\% in terms of model dimensions with respect to the MP model. These complexity reduction results were achieved because the MP model is an oversized model since it uses the same high nonlinearity order in all of the memory branches. In the DPD model with single-feedback, 11th odd-order nonlinearity and memory depth of three were employed, as reported in [3]. Hence, the number of FLOPs and coefficients are slightly increased to 210 and 24, respectively, as compared with the proposed model.

Table 3. Comparison of DPD models’ computational complexity and number of coefficients

\begin{tabular}{ccc}
\hline DPD model & Number of model coefficients & No. of FLOPs \\
\hline MP [2] & $K(Q+1)=28$ & $10+2(K-1)+8 K(Q+1)-2=244$ \\
DPD model [3] & $K_{\text {odd order }}(Q+1)=24$ & $10+2(K-1)+8 K(Q+1)-2=210$ \\
Proposed model & $(M+1)+(L+1)+(K-2)(Q+1)=23$ & $10+2(K-1)+8[(M+1)+(L+1)+(K-2)(Q+1)]-2=204$ \\
\hline
\end{tabular}

In summary, the proposed model outperforms both the DPD model presented in [3] in terms of ACLR performance by $5 \mathrm{~dB}$ with a comparable computational complexity and MP model in terms of linearity performance by $10.8 \mathrm{~dB}$ with a complexity reduction of almost $17 \%$ in the FLOPs as well as a reduction of $18 \%$ in the number of model coefficients. These results demonstrates that a high linearity performance was achieved while the computational complexity of the proposed DPD model was minimized. Consequently, these improvements will lead to reduction in transmitter power consumption and also reduction in hardware resources required for DPD implementation.

\section{CONCLUSION}

In this paper, a DPD model with low-complexity was proposed for linearization of PAs. The proposed model consists of three parallel dynamic branches using a linear memory effects, a low-order nonlinearity memory effects, and a high-order nonlinearity memory effects functions. The linearity performance of the proposed model was validated using a class AB power amplifier driven by a single-carrier WCDMA signal and compared to the MP model as well as the DPD with single-feedback model. The experimental results clearly illustrated that the proposed model had a better performance than the previous models in reducing the ACLR by $10.8 \mathrm{~dB}$ and $5 \mathrm{~dB}$, respectively. Moreover, the computational complexity of the proposed model was reduced by $17 \%$ and $18 \%$ in terms of FLOPs and number of model coefficients, respectively, as compared to the complexity of the MP model. The enhanced performance and complexity reduction of the proposed predistorter are expected to improve the PA efficiency and reduce the overall power consumption in transmitters, respectively.

\section{REFERENCES}

[1] Gharaibeh K. M., “Nonlinear Distortion in Wireless Systems: Modeling and Simulation with Matlab,” John Wiley and Sons Ltd., United Kingdom, 2012.

[2] Ding L., et al., "A Robust Digital Baseband Predistorter Constructed Using Memory Polynomials,” IEEE Trans. Comm, vol/issue: 52 (1), pp. 159-165, 2004.

[3] Cai S., et al., "Digital predistortion based on single-feedback method and indirect learning structure," Analog Integ. Circuits and Sig. Process, vol/issue: 75(1), pp. 125-131, 2013.

[4] Liu Y. J., et al., "A Robust Augmented Complexity- Reduced Generalized Memory Polynomial for Wideband RF Power Amplifiers,” IEEE Trans. Indus. Elect, vol/issue: 61(5), pp. 2389-2401, 2014.

[5] Morgan D. R., et al., "A Generalized Memory Polynomial Model for Digital Predistortion of RF Power Amplifiers,” IEEE Trans. Sig. Process, vol/issue: 54(10), pp. 3852-3860, 2006.

[6] Landin P. N., et al., "Two novel memory polynomial models for modeling of RF power amplifiers," Int. J. Microwave and Wireless Tech., vol. 7, pp. 19-29, 2015. 
[7] K. P. Lee, et al., “A Polynomial Digital Pre-Distortion Technique Based on Iterative Architecture,” International Journal of Electrical and Computer Engineering, vol/issue: 6(1), pp. 106-112, 2016.

[8] Cui H. and Zhao X. M., "A novel NN-predistorter learning method for nonlinear HPA,” Wireless Personal Comm., vol/issue: 63(2), pp. 469-482, 2012.

[9] Mkadem F. and Boumaiza S., "Physically inspired neural network model for RF power amplifier behavioral modeling and digital predistortion,” IEEE Trans. Microw. Theory Tech., vol/issue: 59(4), pp. 913-923, 2011.

[10] Swaminathan J. N. and Kumar P., "Design of Efficient Adaptive Predistorter for Nonlinear High Power Amplifier," Wireless Personal Comm, vol/issue: 82(2), pp. 1085-1093, 2015.

[11] Younes M. and Ghannouchi F. M., "Generalised twin-box model for compensation of transmitters radio frequency impairments,” IET Comm, vol/issue: 8(4), pp. 413-418, 2014.

[12] Zhu A., et al., "Dynamic deviation reduction-based Volterra behavioral modeling of RF power amplifiers," IEEE Trans. Microw. Theory Tech., vol/issue: 54(12), pp. 4323-4332, 2006.

[13] M. F. Siddique, et al., "Design and Simulation of Cascaded Class-A Microwave Power Amplifier," International Journal of Electrical and Computer Engineering, vol/issue: 3(5), pp. 635-639, 2013.

[14] Moon J. and Kim B., "Enhanced Hammerstein behavioral model for broadband wireless transmitters," IEEE Trans. Microw. Theory Tech., vol/issue: 59(4), pp. 924-933, 2011.

[15] Rawat M., et al., "Generalized Rational Functions for Modeling and Digital Predistortion of Broadband Wireless Transmitters,” IEEE Trans. Instrumen. Meas, vol/issue: 63(2), pp. 485-498, 2014.

[16] Eun C. and Powers E. J., "A new Volterra predistorter based on the indirect learning architecture," IEEE Trans. Sig. Process, vol/issue: 45(1), pp. 223-227, 1997.

[17] Liu Y. J., et al., "Modified least squares extraction for Volterra-series digital predistorter in the presence of feedback measurement errors,” IEEE Trans. Microw. Theory Tech., vol/issue: 60(11), pp. 3559-3570, 2012.

[18] Ding L. and Zhou G. T., "Effects of even-order nonlinear terms on power amplifier modeling and predistortion linearization,” IEEE Trans. Vehicular Tech., vol/issue: 53(1), pp. 156-162, 2004.

[19] Tehrani A. S., et al., "A comparative analysis of the complexity/accuracy tradeoff in power amplifier behavioral models,” IEEE Trans. Microw. Theory Tech., vol/issue: 58(6), pp. 1510-1520, 2010.

\section{BIOGRAPHIES OF AUTHORS}

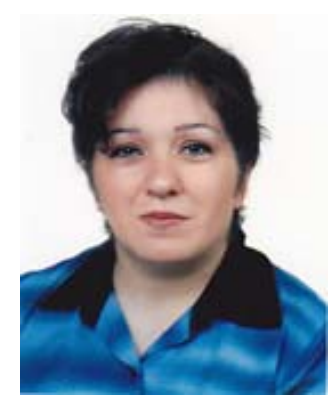

Siba Monther Yousif received B.Sc. degree in Electronic and Communication Engineering and M.Sc. degree in Electronic Engineering from the University of Technology, Iraq, in 1992 and 2007, respectively. She has 12 years of experience in designing and developing electronic circuits for wireless communication systems. She joined the Department of Electronic and Communication Engineering in Al-Nahrain University, Iraq, as an assistant lecturer in 2007. She is currently a Ph.D. candidate in the Department of Electrical and Electronic Engineering in University Putra Malaysia (UPM), Malaysia. She is an IEICE member and a consultant member of Iraqi Engineers Union. Her research interests are in the areas of linearization of power amplifiers and wireless communication systems.

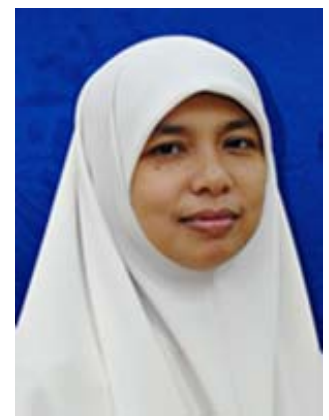

Roslina Mohd Sidek received B.Sc. degree in Electrical Engineering from the George Washington University, Washington D.C, USA in 1990. She received M.Sc. degree in Microelectronics Systems Design and Ph.D. degree in Microelectronics from University of Southampton, UK. She joined Universiti Putra Malaysia (UPM) as a lecturer in 1999. She is currently an associate professor in the Department of Electrical and Electronic Engineering, UPM. Her research interests and her publications are in the areas of semiconductor devices and fabrication, integrated circuit (IC) design and test, and nanoelectronics. She is an IEEE member and has joined IEEE Electron Devices Society and IEEE Circuits and Systems. 

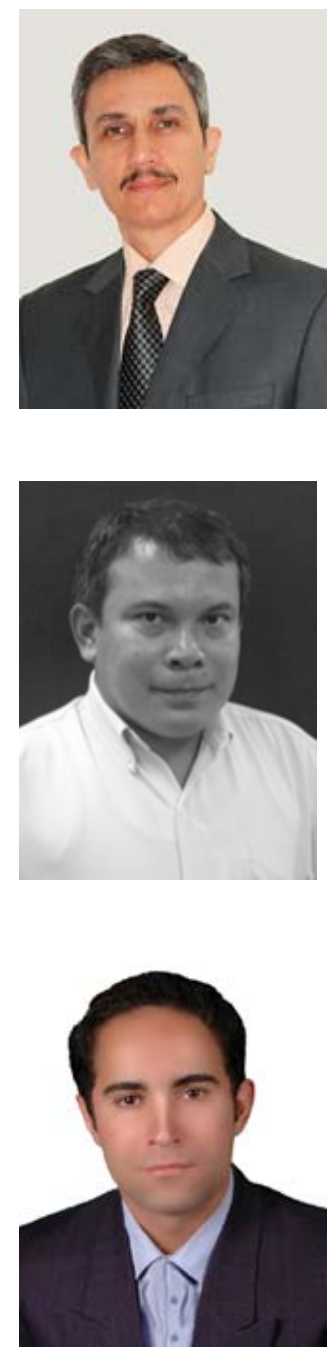

Anwer Sabah Mekki received B.Sc. degree in Electronic and Communication Engineering from University of Technology (U.O.T), Baghdad, Iraq in 1992. He worked in private sectors in designing electronic circuits for wireless control systems, maintenance of computer numerical control machines $\mathrm{CNC}$ and designing infrared sensors for radar applications. He was the head of maintenance department of ICCB/Iraqi Consultants and Construction Bureau in 2004. Then, he was the head of Electronic and Communication Department of Alardh Alkhadraa Company in 2009. He is currently a Ph.D. candidate in the Institute of Advanced Technology in University Putra Malaysia (UPM), Malaysia. He is an IEICE member and a consultant member of Iraqi Engineers Union. He is interested in sensor circuits, microstrip techniques, and wireless communication systems.

Nasri Sulaiman received B.Eng. degree from University of Putra Malaysia in 1994, M.Sc. degree from University of Southampton, UK in 1999, and Ph.D. degree from University of Edinburgh, UK in 2007. He has more than 110 journal and conference papers. He is currently a senior lecturer in the Department of Electrical and Electronic Engineering at University Putra Malaysia, UPM. His research interests are in the areas of signal processing and Evolvable Hardware (EHW). Also, he is the head of control and automation laboratory as well as an expert supervisor of projects at Iranian Institute of Advanced Science and Technology, Shiraz, Iran.

Pooria Varahram received B.Sc. degree in Electrical and Electronics Engineering from the Khaje Nasir University, Tehran, Iran in 2002. He received M.Sc. and Ph.D. degrees in Wireless Communications from Tarbiat Modares, Tehran, Iran, and University Putra Malaysia, in 2005 and 2010, respectively. He was a postdoctoral fellow in University Putra Malaysia till July 2012. He was senior lecturer at University Putra Malaysia from 2013 to 2015. He is currently a senior postdoctoral researcher at Callan Institute, Maynooth University, Ireland. He is a member of IEEE since 2010. He has more than 10 years of experience in designing and developing a range of electronic and telecommunication related projects. His research interests are PAPR reduction in OFDM systems, Linearization of power amplifiers, and microwave power amplifier design. 